\title{
Effects of Chinese herbal medicine Pugongying for reducing the application of antibiotics in breastfeeding women with acute mastitis: study protocol of a randomized, active-controlled, outcome assessor-blinded trial
}

\section{Xinyan Jin ( 20170941260@bucm.edu.cn )}

Beijing University of Chinese Medicine https://orcid.org/0000-0001-7367-5592

Chunli Lu

Centre for Evidence-based Chinese Medicine of Beijing Unitersity of Chinese Medicine

Jianping Liu

Centre for Evidence-based Chinese Medicine of Beijing University of Chinese Medicine

Yingyi Fan

Third Affiliated Hospital of Beijing University of Chinese Medicine

Jinhe Xiao

Third Affiliated Hospital of Beijing University of Chinese Medicine

Chao Gao

Third Affiliated Hospital of Beijing University of Chinese Medicine

Xiaohua Pei

Eighth Affiliated Hospital of Beijing University of Chinese Medicine https://orcid.org/0000-0003-30438874

\section{Research Article}

Keywords: Cefdinir, Herba Taraxaci, Mastitis, Pugongying granule, Traditional Chinese medicine

Posted Date: August 12th, 2021

DOl: https://doi.org/10.21203/rs.3.rs-408139/v1

License: (c) (1) This work is licensed under a Creative Commons Attribution 4.0 International License.

Read Full License 


\section{$1 \quad$ Title}

2 Effects of Chinese herbal medicine Pugongying for reducing the application of antibiotics in breastfeeding

3 women with acute mastitis: study protocol of a randomized, active-controlled, outcome assessor-blinded trial.

\section{Names protocol contributors}

5 Xin-yan Jin, Chun-li Lu, Jian-ping Liu, Ying-yi Fan, Jin-he Xiao, Chao Gao, Xiao-hua Pei

\section{Abstract} support its effectiveness and safety. December 18th 2018. 


\section{Keywords}

29 Cefdinir, Herba Taraxaci, Mastitis, Pugongying granule, Traditional Chinese medicine.

\section{Administrative information}

31 Note: the numbers in curly brackets in this protocol refer to SPIRIT checklist item numbers. The order of the

32 items has been modified to group similar items (see http://www.equator-network.org/reporting-

33 guidelines/spirit-2013-statement-defining-standard-protocol-items-for-clinical-trials/).

\begin{tabular}{|c|c|}
\hline Title $\{1\}$ & $\begin{array}{l}\text { Effects of Chinese herbal medicine Pugongying for reducing the } \\
\text { application of antibiotics in breastfeeding women with acute mastitis: } \\
\text { study protocol of a randomized, active-controlled, outcome assessor- } \\
\text { blinded trial }\end{array}$ \\
\hline Trial registration $\{2 a$ and $2 b\}$. & $\begin{array}{l}\text { ClinicalTrials.gov, ID: NCT03756324. Registered on December 18th } \\
2018, \\
\text { https://www.clinicaltrials.gov/ct2/show/NCT03756324?cond=Acute+m } \\
\text { astitis\&draw=2\&rank=1 } \\
\text { Item 2b: Not applicable, as the study is not registered on World } \\
\text { Health Organization Trial Registration Data Set. }\end{array}$ \\
\hline Protocol version $\{3\}$ & Version 1.0. Date: May, 2018. \\
\hline Funding $\{4\}$ & $\begin{array}{l}\text { This trial is supported financially by the } 2018 \text { Capital's Funds for } \\
\text { Health Improvement and Research (CFH 2018-7032). }\end{array}$ \\
\hline Author details $\{5 a\}$ & $\begin{array}{l}\text { Xin-yan Jin: Third Affiliated Hospital, Beijing University of Chinese } \\
\text { Medicine, Beijing China. 20170941260@bucm.edu.cn }\end{array}$ \\
\hline
\end{tabular}




\begin{tabular}{|c|c|}
\hline & $\begin{array}{l}\text { Chun-li Lu: Centre for Evidence-based Chinese Medicine, Beijing } \\
\text { University of Chinese Medicine, Beijing, China. } \\
\text { annyzhenni@163.com } \\
\text { Jian-ping Liu: Centre for Evidence-based Chinese Medicine, Beijing } \\
\text { University of Chinese Medicine, Beijing, China. liujp@bucm.edu.cn } \\
\text { Ying-yi Fan: Third Affiliated Hospital, Beijing University of Chinese } \\
\text { Medicine, Beijing, China. fan38898901@126.com } \\
\text { Jin-he Xiao: Third Affiliated Hospital, Beijing University of Chinese } \\
\text { Medicine, Beijing, China. } \underline{405873323 @ q q . c o m} \\
\text { Chao Gao: Third Affiliated Hospital, Beijing University of Chinese } \\
\text { Medicine, Beijing, China. } \underline{942846967 @ q q . c o m} \\
\text { Xiao-hua Pei*: Eighth Affiliated Hospital, Beijing University of Chinese } \\
\text { Medicine, Xiamen City, Fujian Province. pxh 127@163.com }\end{array}$ \\
\hline $\begin{array}{l}\text { Name and contact information for } \\
\text { the trial sponsor }\{5 \mathrm{~b}\}\end{array}$ & $\begin{array}{l}\text { Investigator initiated clinical trial: Xiao-hua Pei (Principal Investigator) } \\
\text { pxh127@163.com. }\end{array}$ \\
\hline Role of sponsor $\{5 c\}$ & $\begin{array}{l}\text { This is an investigator initiated clinical trial. Therefore, the sponsor is } \\
\text { not involved in study design, collection, analysis and interpretation of } \\
\text { data, writing of the report and submission of the manuscript for } \\
\text { publication. }\end{array}$ \\
\hline
\end{tabular}

Introduction

\section{Background and rationale $\{6 \mathbf{a}\}$}

36 Acute mastitis is a common problem in lactating women.[1] It is defined that part of one breast becomes red, 37 painful, swollen and hard, sometimes with common symptoms of fever and malaise.[1] The prevalence rates 38 of acute mastitis in breastfeeding women range from $2 \%$ to $33 \%$ according to previous mastitis prevalence 39 data.[1] Acute mastitis may produce overwhelming acute symptoms that causes women to consider to stop 
breastfeeding, or health-care workers will advise women who are administered antibiotics therapy to stop

41 breastfeeding, both of which will result in breastfeeding failure and the infants lose their optimal nutrition.[1-2] What's more, acute mastitis also can bring severe complications such as breast abscess, and occasionally be fatal if inadequately treated.[1] These conditions can lead to a considerable burden of disease and involve substantial costs.[3] Previous studies have indicated that Gram-positive staphylococci is the main pathogenic bacteria in acute mastitis.[4-7] With the increasing application of metagenomic sequencing technology in milk microecology field, the research methods of acute mastitis pathogenic bacteria have gradually turned from the isolation and cultivation of pathogens to equilibrium between microorganism. And these studies suggest that two theories may explain the occurrence of acute mastitis. One is that Staphylococcus and Corynebacterium could not be inhibited by commensal bacteria, the other is lower microbial diversity in milk, with increased abundance of conditioned pathogens and depletion of commensal obligate anaerobes.[8-10] The pathogenesis of acute mastitis has not been thoroughly described, but most investigators prefer that dysbiosis of milk microbiome and/or bacterial infections contribute to the condition. Non-pharmacological measures that have shown promise include effective milk removal, rest, adequate fluids and nutrition, and cold packs application to the breast.[2] Pharmacological measures that have been recommended include analgesia (ibuprofen) and antibiotics. $[2,11]$ The role of probiotic in prevention and treatment is under determined.[2] The preferred antibiotics are usually penicillinase-resistant penicillins, such as dicloxacillin or flucloxacillin, or as recommended by local antibiotic sensitivities.[2] In China, cephalosporin is widely used in clinical practice. However, mistaking antibiotic can affect the physical function, even infants breastfeed in clinical observation.

Chinese herbal medicine is one of the most common traditional interventions in China.[12] Acute mastitis belongs to the syndrome of heat stagnation in both liver and stomach in Traditional Chinese Medicine (TCM) theory. The stomach receives food and drink, and the liver governs free flow of qi. The stagnation qi of stomach is due to dietary irregularities, and the stagnation of liver is due to the failure of liver to disperse qi resulted from emotional depression, which can both result in qi depression transforming into fire. In TCM rationale, breasts belong to stomach meridian and nipples belong to liver meridian.[13] Thus, lactating women with acute mastitis manifest part of usually one breast becoming red, painful, swollen and hard. 
approved by China Food and Drug Administration (CFDA) in 2015. The main ingredient of Pugongying

Granule is Pugongying herbs. The action of Pugongying is to clear liver and stomach heat. Some

71 pharmacological studies have showed that Pugongying has a broad spectrum of antimicrobial activity, and can balance microorganism.[14-17] At the same time, Pugongying can also promote the production of milk and maintain the patency of milk well. [18] The previous clinical trial (unpublished) that had been conducted in the Third affiliated hospital of Beijing University of Chinese Medicine, has demonstrated that Chinese herbal medicine can act better than cefdinir in the time to resolution of fever and visual analogue scale (VAS) scores of breast pain. Although Pugongying has demonstrated positive effects on acute mastitis in clinical practice, rigid validation using a randomized controlled trial remains the best way to examine the effects of Pugongying in lactating women with acute mastitis.

\section{Objectives $\{7\}$}

We hypothesize that Pugongying have positive effects on fever-resolution, less breast pain and massdissipating, and to some extent, it can reduce the application of cefdinir.

\section{Trial design $\{8\}$}

This trial is a Principle Investigator-initiated, three-arm, multicenter, randomized, active-controlled, outcome assessor-blinded, parallel assignment clinical trial in which 306 participants will be assigned to three groups in 1:1:1 ratio with Pugongying alone, cefdinir alone or combination of Pugongying and cefdinir. The investigators plan to allocate a 3-day treatment and 3-day follow-up to participants. Two visits will be scheduled for each participant: baseline, day-3. At the day-6, the investigators will follow the participants up by telephone or Wechat (a social media used in China).

\section{Methods: Participants, interventions and outcomes}

\section{Study setting $\{9\}$}

91 Participants will be recruited from clinics in three hospitals: Third Affiliated Hospital, Beijing University of 92 Chinese Medicine, Beijing Hospital of Traditional Chinese Medicine, and Tongzhou Maternal \& Child Health Hospital of Beijing, which are all located in Beijing, China.

\section{Eligibility criteria $\{10\}$}


96 - Lactating women who have intention to breastfeed child;

97 - Within 72 hours after the onset of symptoms and/or signs (body temperature $\geqq 37.2^{\circ} \mathrm{C}$ with at least one

98 symptoms, such as red, painful, swollen and hard of part of breast), and the ultrasound examination

99 indicates there is no mammary abscess;

$100-$ The body temperature is higher than $37.2^{\circ} \mathrm{C}$ but lower than $41^{\circ} \mathrm{C}$;

101 - The VAS scores of breast pain (range from $0-10$ ) $\geqq 4$;

102 Willing and able to comply with protocol requirements and provide informed consent.

103 Exclusion criteria

104 - Having other breast disease that hinders or prevents breastfeeding;

105 - Having taken therapeutic drugs for this episode of acute mastitis;

106 Known allergic to penicillin and cephalosporin;

107 Participants with mental disorders, seizure disorders, or cognitive dysfunction;

108 - Presence of any other pre-existing chronic infection requiring medical therapy;

109 - Having any history of chronic liver disease, or any active lung, heart or renal diseases requiring regular 110 medication.

111 The three hospitals are equipped with Breast-Disease Clinics and there are enough patients with acute 112 mastitis to ensure the implementation of this trial.

\section{Who will take informed consent? $\{26 \mathrm{a}\}$}

114 The doctors who will obtain Informed Consent From (ICF) from potential trial participants are licensed and 115 have obtained Good Clinical Practice (GCP) certificates in each hospital. All participants should give written 116 ICF prior to participating the study. The doctors should inform the participants of the protocol, objective, 117 rights and interests, possible risks of the study, study confidentiality and related compensation. And the 
118 participants should be assured that they can quit the study any time.

119 Additional consent provisions for collection and use of participant data and 120 biological specimens $\{26 \mathrm{~b}\}$

121 The participants in Pugongying group and cefdinir group will sign another ICF which contains the item about 122 milk samples collection for further genetic analysis. Three milk samples will be collected on the baseline day 123 (day-0) and day-3. All samples will not be preserved.

\section{Interventions}

\section{Explanation for the choice of comparators $\{6 \mathbf{b}\}$}

126 The recommended pharmacological measure of acute mastitis is mainly antibiotics.[2, 12] The preferred 127 antibiotics are usually penicillinase-resistant penicillins, such as dicloxacillin or flucloxacillin, or as 128 recommended by local antibiotic sensitivities.[2] Cefdinir is the 3rd generation of cephalosporin. Due to its 129 few adverse events, weak toxicity, broad anti-microbial spectrum and less prone to drug tolerance, it has 130 been widely applied to mastitis treatment in China. Thus, this trial chooses it as active-controlled 131 intervention.

\section{Intervention description $\{11 \mathrm{a}\}$}

133 In clinics, participants will be randomly assigned to the Chinese patent medicine group (CPM), combination 134 of Chinese patent medicine and Antibiotics cefdinir capsule group (CPM \& ACC), or Antibiotics cefdinir 135 capsule group (ACC).

136 For participants in CPM group, Pugongying should be taken $15 \mathrm{~g}$ three times a day for 3 days. For CPM \& 137 ACC participants, Pugongying should be taken $15 \mathrm{~g}$ three times a day for 3 days and cefdinir should be taken $1380.1 \mathrm{~g}$ three times a day in the first two days. For participants in ACC group, cefdinir should be taken $0.1 \mathrm{~g}$ 139 three times a day for 3 days.

140 Pugongying is named as Pugongying Granule in China and produced by Kunming Pharmaceutical Factory 141 co. LTD. The form of the drug is granule, and the participants will take the drug after dissolved. Cefdinir is 142 also named as cefdinir capsule and produced by Astellas Pharma Inc. The form of the drug is capsule, and 143 participants will orally take the capsules. The investigators will follow them up for 3 days after 3-day drug 
administration.

145 All participants will receive education, including dietary, emotional regulation and the knowledge of 146 breastfeeding. The investigators will encourage participants to remove milk effectively.

147 Criteria for discontinuing or modifying allocated interventions $\{11 \mathrm{~b}\}$

148 The participants with body temperature above $41^{\circ} \mathrm{C}$ in the 3-day treatment period will be recommended to 149 withdraw from the trial and receive intravenous drip antibiotics therapy. If the participants have preference 150 for particular intervention and would not like to continue receiving assigned intervention, they can withdraw 151 from the trial at any time. In the above situations, the investigators will try their best to obtain the participants' 152 data when they withdraw from the trial.

153 During 3-day treatment, if B-ultrasound hints that there is a mammary abscess, the doctor will discuss with 154 the participants and additionally perform the surgery to prevent them from severe complications if necessary. 155 The participants in CPM group with body temperature above $39^{\circ} \mathrm{C}$ will be administered by twice dose of 156 Pugongying.

\section{Strategies to improve adherence to interventions $\{11 \mathrm{c}\}$}

158 Before the beginning of enrollment, the doctors will inform them about high recurrence rate of acute mastitis 159 and the necessity of 3-day drugs administration. And the doctors can assess the disease condition by related 160 laboratory tests and examinations on day-3 to ensure the interests of all enrolled participants. Finally, the 161 investigators will distribute $120 \%$ intervention drugs and require the participants to return the drugs at day-3.

\section{Relevant concomitant care permitted or prohibited during the trial $\{11 \mathrm{~d}\}$}

163 Education, such as dietary, emotional regulation and the knowledge of breastfeeding, and effective milk 164 removal are permitted. Other drugs for treating acute mastitis, such as ibuprofen and probiotic are prohibited.

\section{Provisions for post-trial care $\{30\}$}

166 Provisions for post-trial care are not applicable. Laboratory tests, examinations and treatments for 167 concomitant diseases or complications are not provided freely by the sponsor, which will be communicated 168 to the participants as they sign the informed consent. 


\section{Outcomes $\{12\}$}

170 Primary outcome measures

171 Primary outcome measures are the common chief complaints which have strong relevance to acute mastitis.

172 - Resolution of fever: Body temperature will be measured by mercury thermometer and recorded on the

173 prepared card by participants. The temperature reduces to $37.2^{\circ} \mathrm{C}$ or more below, assessing as the normal

174 temperature. And the normal temperature lasts for at least 24 hours, considered as fever-resolution. To 175 evaluate onset time and the temperature changing from baseline to the end of 3-day treatment, participants 176 will be encouraged to measure body temperature every 4 hours for 3 days, specifically on 2:00 am, 6:00 am, $177 \quad 10: 00 \mathrm{am}, 14: 00 \mathrm{pm}, 18: 00 \mathrm{pm}, 22: 00 \mathrm{pm}$.

178 - VAS sores of breast pain: Breast pain will be self-reported by participants and recorded. The VAS is used 179 to assess breast pain. The scale has been tested in the investigator's previous trial. 0 score indicates "no 180 uncomfortable feeling". 1-3 indicates "mild uncomfortable feeling". 4-6 indicates "moderate uncomfortable 181 feeling". 7-10 indicates "severe uncomfortable feeling". To evaluate the changing from baseline to the end of 182 3-day treatment, participants will assess breast pain every 8 hours for 3 days, specifically measured on 6:00 183 am, 14:00 pm, 22:00 pm.

184 - The size of the breast mass: The mass will be manually outlined by outcome assessors with a measurement film which have registered the patent (Patent No. ZL-2010-2-0172672.8). [19]To evaluate the changing from baseline to the end of 3-day treatment, the mass will be measured at baseline and at the end of the treatment, total 2 times.

Secondary outcome measures

- The patency of milk: The outcome will be measured by the outcome assessor. The $0-3$ scores are used to 190 describe the patency of milk from no stagnation to severe stagnation. 0 indicates that there is no stagnation

191 with breast and milk spurts out by slightly pressure; 1 indicates mild stagnation and milk flows by more

192 pressure; 2 indicates moderate stagnation and milk flows by much more pressure; 3 indicates there is severe 193 stagnation and no milk flows. To evaluate the changing, the outcome will be evaluated at baseline and at the 194 end of the treatment, total 2 times. 
- TCM symptoms scores: The assessment criteria refer to Standard of diagnosis and treatment in TCM

196 symptoms (2016 version, released by State Administration of Traditional Chinese Medicine of the People's

197 Republic of China, http://www.stctcm.com/STCM/LineAnnouncement/899.htm). The criteria are specifically

198 used to assess the holistic physical status of participants and will be measured by outcome assessors. To

199 evaluate the changing, the outcome will be evaluated at baseline and at the end of the treatment, total 2

200 times.

201 -White blood cell count: Measured by the routine blood test. To evaluate the changing, it will be tested at 202 baseline and at the end of 3-day treatment, total 2 times.

203 - Percentage of neutrophil: Measured by the routine blood test. To evaluate the changing, it will be tested at 204 baseline and at the end of 3-day treatment, total 2 times.

205 - C-reactive protein: Measured by the routine blood test. To evaluate the changing, it will be tested at 206 baseline and at the end of 3-day treatment, total 2 times.

207 - The quantity of intervention drugs and the incidence of surgery: After 3-day drug administration, the 208 participants will undergo the clinical evaluation at the 2 nd visit and the doctor will recommend whether 209 participants need further treatment. If B-ultrasound shows there is a mammary abscess, the doctor will 210 perform surgery, or prescribe cefdinir or Pugongying based on liquefaction and size of the abscess. Then the 211 investigators will record the quantity of drugs and the incidence of surgery.

212 - Relapse of acute mastitis: After 3-day treatment, the investigators will follow the participants up for another 2133 days to figure out the incidence of acute mastitis relapse. The investigators will call the participants to 214 inquiry their conditions (body temperature and breast pain).

215 - Safety assessments: To assess the safety of the interventions, the investigators will perform the following 216 tests on participants at baseline and the end of 3-day treatment: routine blood, liver and renal function and 217 electrocardiography. All abnormal values are defined based on reference values.

\section{Participant timeline $\{13\}$}

219 Table 1 shows the participant timeline (Please see Table 1 at the end of the document). 
221 We analyze all available data from the pilot study on breast-pain VAS scores in lactating women with acute mastitis (unpublished) in Third Affiliated Hospital of Beijing University of Chinese Medicine, and the suggestions from the practitioners to calculate sample size. In this trial, sample size formula in the $4^{\text {th }}$ edition clinical epidemiology is used as reference.[20] In terms of sample size, there is in excess of a 95\% power and a (2-side) $10 \%$ significance level in detecting treatment differences. The standard deviations are 0.81 in in ACC, respectively.

$$
\mathrm{n}=\frac{\Psi^{2}\left(\sum_{\mathrm{j}=1}^{\mathrm{k}} \sigma_{\mathrm{j}}^{2} / \mathrm{k}\right)}{\sum_{\mathrm{j}=1}^{\mathrm{k}}\left(\overline{\mathrm{X}_{\mathrm{j}}}-\overline{\mathrm{X}}\right)^{2} /(\mathrm{k}-1)}
$$

229 As a result, $\mathrm{j}-1$

$\Psi_{0.55 .0 .10 .2 . \infty}=2.52$. Bring the results into the formula above, $\mathrm{n}=\frac{2.52^{2}(2.0437 / 3)}{0.1014 / 2} \approx 85$

232 attrition rate, the sample size of each group is 102 and 306 patients will be recruited in total.

\section{Recruitment $\{15\}$}

234 The costs of intervention drugs (Pugongying and cefdinir), laboratory tests (routine blood, routine urine) and 235 examinations (breast B-ultrasound and electrocardiogram) will be provided by the sponsor.

236 Assignment of interventions: allocation

\section{Sequence generation $\{\mathbf{1 6} \mathbf{a}\}$}

238 The sequence of randomization (1:1:1) will be generated by JHX with a computer program (Excel). 
240 The randomized number and allocation details will be sealed in opaque envelopes.

241 Implementation $\{16 c\}$

$242 \mathrm{JHX}$ will generate the allocation sequence. The doctors who in clinics will enroll participants. And the 243 investigators (e.g. JHX and XYJ) will assign participants to interventions.

\section{Assignment of interventions: Blinding}

\section{Who will be blinded $\{17 \mathrm{a}\}$}

246 The trial is an outcome assessor-blinded, data collector-blinded and data analyst-blinded study. Treatment 247 allocations will be concealed from the data analysts by group 1, 2 and 3. The data-collectors (e.g. CG) and 248 outcome assessors (e.g. nurses) blinded about intervention allocations will record the data and evaluate the 249 mass size, patency of milk, TCM symptoms and relapse. Participants preference can have minor influence 250 on body temperature, white blood cell count, the percentage of neutrophil and C-reactive protein.

\section{Procedure for unblinding if needed $\{17 \mathrm{~b}\}$}

252 Not applicable. In this trial, participants and doctors will not be blinded, therefore there is no unblinding 253 procedure.

\section{Data collection and management}

\section{Plans for assessment and collection of outcomes $\{18 \mathrm{a}\}$}

256 The investigators (JHX, XYJ) have studied for the use of measurement films at The People's Hospital of

257 Liaoning Province on 5th May 2019. XHP, YYF, XYJ and JHX have implemented the project 'The clinical 258 effects of Shaoyao Gualou Gancao decoction combined with Xiaozhong Zhitong patches on the initial stage 259 of acute mastitis: A randomized control trial (unpublished)' and have a good understanding of acute mastitis-

260 related measurement methods and data collection. $\mathrm{JHX}$ and XYJ have trained the outcome assessors and 261 data collectors in three hospitals and will not participate in these works. Laboratory tests will be performed in 262 clinical laboratory of each hospital. All abnormal values are defined based on reference values. 
263 Plans to promote participant retention and complete follow-up $\{18 \mathrm{~b}\}$

264 During intervention period and follow-up period, the investigators will communicate with all enrolled 265 participants by telephone or Wechat to obtain the information of the participants' conditions (e.g. body 266 temperature, breast pain). If the participants discontinue or deviate from the study, the investigators will 267 persuade them to receive laboratory tests and examinations to assess their disease conditions and protect 268 their interests.

\section{Data management $\{19\}$}

270 All data will be input and checked by two statisticians using EpiData 3.1.

\section{Confidentiality $\{27\}$}

272 All participants' personal information will be confidential to the extent permitted by Chinese laws. The 273 samples of enrolled participants will be identified by study numbers rather than their name. Unless the 274 permission is obtained, information that identifies individuals will not be disclosed to anyone other than 275 members of the study group. The investigators, the supervisor appointed by $\mathrm{CFH}$, the ethics committee and 276 CFDA are allowed to access participants' medical records related to the study to ensure the authenticity and accuracy of the data, but other individual information will not be shared. Case report forms (CRF) will be reserved in cabinet unless investigators allow to open. Electronic data will be input according to the study number and accessed under the permission of investigators. When the results of this study are published, no information about the participants will be disclosed.

281 Plans for collection, laboratory evaluation and storage of biological specimens for genetic or molecular analysis in this trial/future use $\{33\}$

283 Four blood, two urine and three milk samples will be collected on the baseline day (day-0) and day-3. Blood 284 samples will be utilized in laboratory tests, including routine blood test, liver and kidney function test. Blood 285 and urine samples will not be saved. All abnormal values will be evaluated based on reference values. 


\section{Statistical methods}

290 Statistical methods for primary and secondary outcomes $\{20 \mathrm{a}\}$

291 Statistical analysis will be conducted by Centre for Evidence-Based Chinese Medicine, Beijing University of 292 Chinese Medicine. The statistician (not in authorship) will be blinded from the intervention allocations. SPSS 29325.0 statistical software packages will be used to analyze the data. Prior to all analyses, a detailed statistical 294 analysis protocol is developed.

295 Continuous variables will be expressed as median and standard deviations. Three groups will be compared 296 using Analysis of Variance (ANOVA) or Kruskal Wallis test, as appropriate, based on the data distribution.

297 Two groups will be compared using Least Significant Difference (LSD) if there is significant differences 298 between three groups. Dichotomous variables will be expressed as "yes" or "no". Groups will be compared 299 using the chi-square or Fisher's exact test, as appropriate, based on the expected counts. Participants 300 characteristics and past history will be reported and compared between groups. Descriptive statistics will be 301 presented to describe the trial results. A two-sided $\mathrm{P}<0.05$ will be considered statistically significant.

Interim analyses $\{21 b\}$

303 Not applicable. The interim analyses are not planned to conduct.

Methods for additional analyses (e.g. subgroup analyses) $\{20 \mathrm{~b}\}$

Not applicable. Additional analyses are not planned to conduct.

Methods in analysis to handle protocol non-adherence and any statistical methods to handle missing data $\{20 \mathrm{c}\}$

308 The intention-to-treat (ITT) population is defined as the patients who are randomized and receive at least 309 one treatment session. The per-protocol (PP) population is defined as the patients who complete the study 310 and do not have major protocol violations. All analyses will be based on the ITT population and the PP 311 population. The result of the ITT analysis will be compared with that of the PP analysis to check whether the 312 results are consistent.[21] 
313 Plans to give access to the full protocol, participant level-data and statistical code

$314\{31 c\}$

315 The data can be available by the corresponding author with reasonable request.

316 Oversight and monitoring

317 Composition of the coordinating centre and trial steering committee $\{5 \mathrm{~d}\}$

318 CFH will be responsible for quality control and the management will comply with the Administrative

319 Measures of Capital's Funds for Health Improvement and Research published by Beijing Health Commission

320 of the People's Republic (2017 version). The sponsor will commission the third party to monitor the trial

321 avoiding interest conflicts.

Composition of the data monitoring committee, its role and reporting structure $\{21 \mathrm{a}\}$

Data monitoring committee is not applicable. The data will be monitored by the sponsor.

Adverse event reporting and harms $\{22\}$

326 Any serious adverse events will be reported to the principle investigator within 24 hours and recorded in CRF

327 to analyze the relationship between events and intervention. The serious adverse events will be reported to

3282018 Capital's Funds for Health Improvement and Research following GCP guidelines.

329 Frequency and plans for auditing trial conduct $\{23\}$

330 The sponsor will randomly audit some clinical trials and commission the third party to conduct the audit

331 procedures to avoid interest conflicts.

332 Plans for communicating important protocol amendments to relevant parties (e.g.

333 trial participants, ethical committees) $\{25\}$

334 Important protocol amendments will communicate with enrolled trial participants, investigators, ethical 335 committees of Beijing University of Chinese Medicine Third Affiliated Hospital and the sponsor. And then the 
336 investigators will update the protocol on ClinicalTrials.gov.

\section{Dissemination plans $\{31 \mathrm{a}\}$}

338 We will put up a poster and Wechat post via public account to disseminate this trial.

\section{Discussion}

340 The results of this trial are expected to provide convincing evidence that Pugongying is effective and safety

341 for alleviating manifestations of lactating women with acute mastitis, and they could reduce application of 342 cefdinir in clinical practice.

343 As we know, milk is not sterile and has a wide range of microbiome which has important health 344 implications.[22, 23] And the relationship of microbiome in milk is in a dynamic equilibrium. The application of 345 antibiotics may induce dysbiosis of milk microbiome.[24] In TCM theory, "vital qi" is a collective designation

346 for all normal function of the human body and the abilities to maintain health, including the abilities to selfregulation, adaptation to the environment, resistance against pathogens and self-recovery from illness, and “ pathogenic qi" specifies various pathogenic factors. The occurrence of infectious diseases is the result of internal and external factors' interactions. Therefore, strengthening body resistance to eliminate pathogenic factors is fundamental therapeutic principle. In microbiology researches, the bacterial communities 351 maintaining the stability, quantities and orders of microbiome are considered as "vital qi". If the balance is disturbed inducing the dysbiosis of milk, mircobiological colonization resistance and immunity can decline generating "pathogenic qi". Based on the hypothesis, the treatment of infectious diseases should focus on

354 the biological antagonism of microbiome and then eliminate the pathogenic factors.[25] And the intervention, 355 Pugongying can work both theoretically.

356 World health organization (WHO) provides a handbook for mothers, and ABM (The Academy of 357 Breastfeeding Medicine Protocol Committee) also publishes the clinical protocol. The recommendations 358 include pharmacological and non-pharmacological interventions, but they do not include TCM which 359 effectively resolves the symptoms of acute mastitis in practice. Pugongying belongs to Chinese patent medicine. Chinese patent medicine has satisfactory characteristics, such as definite benefit response, 
361 guaranteed safety assessment, and it is convenient to taking, carrying and storage. Proving the

362 effectiveness of Pugongying will be helpful to the generalization of TCM. However, there is not enough

363 evidence for Pugongying based on methodology rigorous clinical trial. In this trial, we conduct a multicenter,

364 randomization, outcome assessor-blinded, parallel assignment clinical trial, with a large sample size to

365 ensure power.

366 There are some limitations of this trial. First, the outcomes of this trial may not be sufficient to evaluate the 367 effectiveness and safety of Pugongying from all angles in the treatment of acute mastitis. However, the core 368 outcome set of mastitis is not available. We try to assess the manifestations of acute mastitis based on 369 clinical experience. Second, we do not use double-blinded design and this may lead to performance bias.

370 But the outcomes measures except the scores of breast pain, are either objective indicators or outcome 371 assessor-evaluation outcomes. The preference of participants and investigators is minimal. Third, the title is 372 "Effects of Chinese herbal medicine Pugongying for reducing the application of antibiotics in breastfeeding 373 women with acute mastitis", but the intervention of this trial is Pugongying which may be not enough to 374 represent Chinese patent medicine. We hope that this trial can preliminarily confirm the effectiveness of 375 Pugongying which is the most representative Chinese patent medicine for the treatment of acute mastitis, 376 and it can be fundamental evidence for future research.

\section{$377 \quad$ Trial status}

378 Protocol version is version-1 in May 2018. The recruitment began on August 19th 2019 and will be 379 completed approximately on December 31st 2020.

\section{Abbreviations}

381 ABM: The Academy of Breastfeeding Medicine Protocol Committee

382 ACC: Antibiotics Cefdinir Capsules group

383 ANOVA: Analysis of Variance

384 CFH: Capital's Funds for Health Improvement and Research 
387 CPM \& ACC: Combination of Chinese Patent Medicine and Antibiotics Cefdinir Capsules group

388 GCP: Good Clinical Practice

389 ICF: Informed Consent From

390 ITT: Intention-to-treat

391 LSD: Least Significant Difference

392 PP: Per-protocol

393 TCM: Traditional Chinese Medicine

394 VAS: Visual Analogue Scale

395 WHO: World health organization

\section{Declarations}

397 - Acknowledgements

398 - Authors' contributions

399 - Funding

400 - Availability of data and material

401 - Ethics approval and consent to participate

402 - Consent for publication

403 - Competing interests

404 - Authors' information (optional)

\section{Acknowledgements}

406 We gratefully acknowledge Professor Hui-juan Cao from Centre for Evidence-Based Chinese Medicine of

407 Beijing University of Chinese Medicine for her technological support on statistical analysis. We also

408 appreciate Ya-ru Xia, Na-na Chen, Yan-ran Zhang and Dong-xiao Zhang for their efforts as clinical research 


\section{Authors' contributions $\{31 \mathrm{~b}\}$}

411 XHP as the Principle Investigator, conceived the study and led the proposal.

$412 \mathrm{XYJ}$ contributed to the study design, drafted the manuscript and participated in the study implementation.

413 CLL assisted in clinical trial registration and participated in the protocol development.

414 JPL was the lead methodologist of this trial.

$415 \mathrm{JHX}$ contributed to development of the proposal and participated in the manuscript.

416 YYF participated in the study design and study implementation.

$417 \quad$ CG assisted in the manuscript.

418 All authors discussed, read, revised the manuscript, and all approved the final manuscript.

419 Funding $\{4\}$

420 This trial is supported financially by the 2018 Capital's Funds for Health Improvement and Research (CFH

421 2018-7032). The sponsor will be responsible for quality control and will not be involved in study design,

422 collection, analysis and interpretation of data, writing of the report and submission of the article for

423 publication. An additional file shows funding information in more details (see Additional file 1).

\section{Availability of data and materials $\{29\}$}

425 After this study is completed, the final trial dataset and statistical codes will be available from the

426 corresponding authors upon reasonable request, except for participants' personal information.

\section{Ethics approval and consent to participate $\{24\}$}

428 Ethics approval of the study has been obtained from the Ethical Committee of Third Affiliated Hospital of 429 Beijing University of Chinese Medicine (number BZYSY-SFKTPJ-1) and Beijing Hospital of Traditional 430 Chinese Medicine (number 2019BL02-028-02) according to Chinese law. Written, informed consent to 431 participate will be obtained from all participants. An additional file shows ethics approval in more details (see 432 Additional file 2). 
434 Not applicable. There is no individual personal data in this manuscript.

\section{Competing interests $\{\mathbf{2 8}\}$}

436 The authors declare that they have no competing interests.

\section{Authors' information (optional)}

\section{Participant Timeline}

439 Table 1 Standard Protocol Items: Recommendations for Interventional Trials (SPIRIT)

\begin{tabular}{|c|c|c|c|c|c|c|}
\hline \multirow[b]{3}{*}{ TIMEPOINT } & \multicolumn{6}{|c|}{ STUDY PERIOD } \\
\hline & \multirow{2}{*}{$\begin{array}{c}\text { Enrolment } \\
0\end{array}$} & \multirow{2}{*}{$\begin{array}{c}\text { Allocation } \\
0\end{array}$} & \multicolumn{3}{|c|}{ Post-allocation } & \multirow{2}{*}{$\begin{array}{c}\text { Close-out } \\
\text { Day-6 }\end{array}$} \\
\hline & & & Day-1 & Day-2 & Day-3 & \\
\hline \multicolumn{7}{|l|}{ ENROLMENT: } \\
\hline Eligibility screen & $\mathrm{X}$ & & & & & \\
\hline Informed consent & $\mathrm{X}$ & & & & & \\
\hline Allocation & & $\bar{X}$ & & & & \\
\hline \multicolumn{7}{|l|}{ INTERVENTIONS: } \\
\hline \multicolumn{7}{|l|}{ CPM group } \\
\hline \multicolumn{7}{|l|}{ ACC group } \\
\hline \multicolumn{7}{|l|}{ CPM \& ACC group } \\
\hline$A C C$ & & & $\leftarrow$ & $\Longrightarrow$ & & \\
\hline ASSESSMENTS: & $\mathrm{X}$ & & & & $\mathrm{X}$ & \\
\hline Body temperature & & $\mathrm{X}$ & $\mathrm{X}$ & $\mathrm{X}$ & $\mathrm{X}$ & $\mathrm{X}$ \\
\hline
\end{tabular}




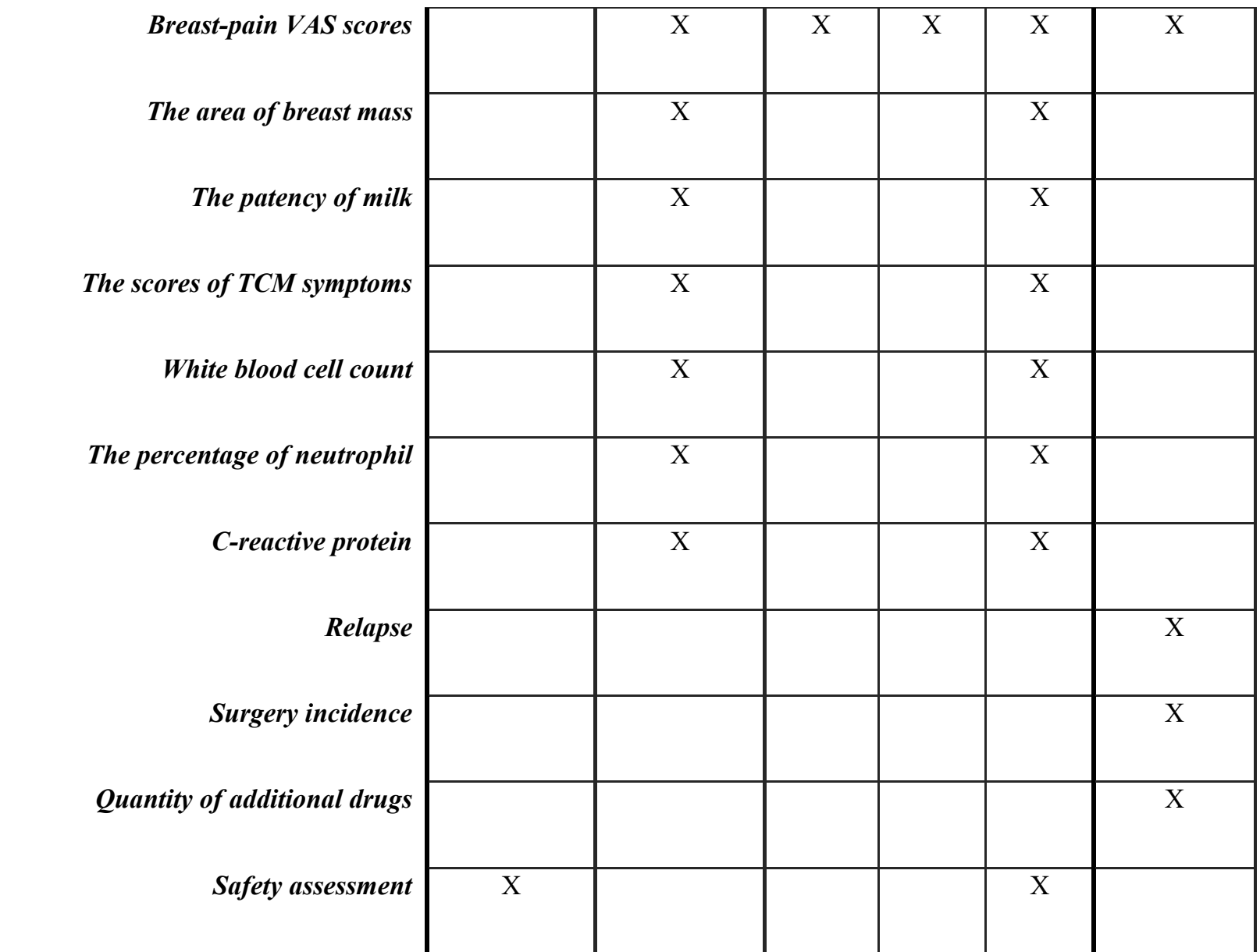

440 Note: CPM- Chinese Patent Medicine group; CPM \& ACC- combination of Chinese Patent Medicine and

441 Antibiotics Cefdinir Capsules group; ACC- Antibiotics Cefdinir Capsules group.

\section{References}

443 [1] World Health Organization. Mastitis: causes and management. Published 2000. WHO Website.

444 http://www.who.int/maternal_child_adolescent/documents/fch_cah_00_13/en/. Accessed December $44512,2019$.

446 [2]Lisa H. Amir and The Academy of Breastfeeding Medicine Protocol Committee, ABM Clinical Protocol \#4:

447 Mastitis, Revised March 2014. Breastfeed Med 2014; 9: 239-43.

448 [3] Murphy DP, SenGupta SK, Muthaiah AC. Benign breast disease in Papua New Guinea. Papua New 449 Guinea Medical Journal 1992;35:101-105.

450 [4] Delgado S, Arroyo R, Martin R, Rodriguez JM. PCR-DGGE assessment of the bacterial diversity of 451 breast milk in women with lactational infectious mastitis. BMC Infect Dis 2008; doi:10.1186/1471-2334-8-51.

452 [5] Esther, Jiménez, Javier, de, Andrés, Marina, et al. Metagenomic Analysis of Milk of Healthy and Mastitis- 
454 [6] Kvist LJ, Larsson BW, Hall-Lord ML, Steen A, Schalén C. The role of bacteria in lactational mastitis and 455 some considerations of the use of antibiotic treatment. Int Breastfeed J 2008;doi:10.1186/1746-4358-3-6. 456 [7] Gan L,Wang T,Min J,Wang YD,Wang YY,Lv G. Breast culture and drug sensitivity analysis in patients 457 with lactating acute mastitis. Chinese Medicine Emergency 2014;10:1912-1914.

458 [8] Heikkila MP, Saris PEJ. Inhibition of Staphylococcus aureus by the commensal bacteria of human milk. J 459 Appl Microbiol 2003;95:471-478.

460 [9] Ma ZS, Guan Q, Ye CX, Zhang CC, Foster JA, Forney LJ. Network analysis suggests a potentially 'evil' 461 alliance of opportunistic pathogens inhibited by a cooperative network in human milk bacterial communities. 462 Sci Rep 2015;doi:10.1038/srep08275.

463 [10] Patel SH, Vaidya YH, Patel1 RJ, Pandit RJ, Joshi CG, \& Kunjadiya AP. Culture independent 464 assessment of human milk microbial community in lactational mastitis. Sci Rep 2017;7:7804.

465 [11] World Health Organization. Hospital care for mothers Guidelines for Management of Common Merternal 466 Conditions. Published 2017. WHO Website.

467 http://www.searo.who.int/entity/maternal_reproductive_health/documents/mpb/en/. Accessed December $46812,2019$.

469 [12] Feng HZ, Yu QX, Ding CY, Zhu Y. The Effectiveness of Traditional Chinese Medicine in Acute Mastitis: 470 A Systematic Review. Zhejiang Journal of Integrated Traditional Chinese and Western Medicine 2019;29: $471 \quad 418-423$.

472 [13] Li YQ, He QH, Liu S, Pan LQ, Song AL, Qin GZ. Surgery of Chinese Medicine. $3^{\text {rd }}$ ed. Beijing: China 473 Press of Traditional Chinese Medicine; 2012.

474 [14] Liu LB, Ping JQ,Gao HF,Zhang XM. Comparison of Antibacterial Effects of Extracted Objects from 475 Different Parts of Dandelion. Journal of Agriculture Science Yanbian University 2010;32: 65-68.

476 [15] Wu XZ, Wang YH, Yang QH. Study on the Antibacterial Effect of Three Flavor Medicine Extracts. 477 Journal of Inner Mongolia Medical College 2008;30: 374-376.

478 [16] Gu YJ, Wang LJ. Extraction of flavonoids from dandelion and its antibacterial properties. Journal of 
480 [17] Shi D, Zhang Y, Effect of dandelion polysaccharide on intestinal microecology of mice. Advances in 481 Microbiology Immunology 2016;44: 49-53.

482 [18] Li HY,Fan HY, Research progress on pharmacological effects of dandelion. China High-tech Zone 483 2018;07: 189-191.

484 [19] Du Gang, Cui Jian-chun, Li Li, et al. Instruction of a breast mass measurement film. Chinese Journal of 485 Practical Surgery. 2011;31Suppl 2:82.

486 [20] Wang Jia-liang, Xu Liang-zhi, Kang De-ying, Wei Qiang, et al. Clinical epidemiology. $4^{\text {th }}$ ed. Shanghai: 487 Shanghai Science and Technology Press;2014.

488 [21] Lu ZH, Dong HL, Huang-fu JW, Fan XJ, Zhao WX, Min S, et al. Effect of dual-acupoint and single489 acupointelectric stimulation on postoperative outcomes in elderly patients subjected togastrointestinal 490 surgery: study protocol fora randomized controlled trial. Trials 2018;19:669.

491 [22] Hunt KM, Foster JA, Forney LJ, Schütte UME, Beck DL, Abdo Z, et al. Characterization of the diversity 492 and temporal stability of bacterial communities in human milk. PLoS One $493 \quad 2011 ; 10.1371 /$ journal.pone.0021313.

494 [23] Sakwinska O, Moine D,Delley M,Combremont S, Rezzonico E, Descombes P, Vinyes-Pares G, et al. 495 Microbiota in Breast Milk of Chinese Lactating Mothers. PLoS One 2016;10.1371/journal.pone.0160856.

496 [24] Langdon A, Crook N, Dantas G. The effects of antibiotics on the microbiome throughout development 497 and alternative approaches for therapeutic modulation. Genome Med 2016;10.1186/s13073-016-0294-z. 498 [25] Wu GL,Yu GY, Lu WW. Discussion on the essence of intestinal microecology in traditional Chinese 499 medicine. Chinese Journal of Traditional Chinese Medicine. 2015. 33: 2586-2588. 